\title{
Strategies for \\ the Visualization \\ of Multiple 2D \\ Vector Fields
}

\begin{abstract}
$\mathbf{T}$
\end{abstract} he study of coherent structures in turbulent boundary layers is an active area of research. Driven by applications including the reduction of turbulent skin-friction drag over aircraft, researchers have long been interested in developing a deeper understanding of the key physical features within turbulent flows. One application of interest is the analysis of multiple scalar and vector distributions and how the interaction of variables contributes to theories of drag and the formation of vortex packets within a turbulent boundary layer. A second application involves examining the correlation between different layers within a numerical simulation of a turbulent channel flow. The analysis of these applications can be greatly facilitated by a

Strategies for effectively visualizing colocated $2 \mathrm{D}$ vector fields enable understanding of key physical structures of one vector field within the context of a related vector field. This article describes the range of effects possible by combining several existing flow visualization techniques for analyzing multiple vector fields. better understanding of the complicated interactions that occur between the vortices that make up the motion.

Important research conducted in the computational physics field focuses on modeling 3D magnetohydrodynamic light supersonic jets in the context of astrophysical jets in galaxy clusters. These high-speed jets propagate distances of over 650,000 light years from their sources, transporting energy and magnetic fields to their surrounding environments. The jet magnetic field is advected along with the flow and is expected to reflect properties of the evolving velocity field. Of particular interest is the extent to which the magnetic and velocity vector fields are spatially aligned and/or orthogonal to one another and the interplay between magnetic field strength and the corresponding velocity structures.

Our research explores strategies for developing effective methods for the visual representation of multiple colocated vector and scalar fields to allow understanding and analyses of each field, both individually and in the context of the other. Mining the knowledge base of previous visualization research yields important findings and insights to assist the research of our specific applications. Using these insights, we can reconstruct,

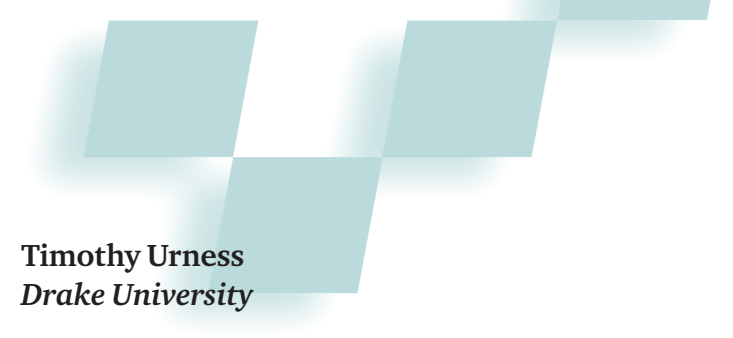

Victoria Interrante, Ellen Longmire, Ivan Marusic, Sean O'Neill, and Thomas W. Jones University of Minnesota

manipulate, and expand upon the existing state of the art to further the knowledge-discovery process related to specific tasks and conditions. Our present efforts focus primarily on the investigation of methods for effectively visualizing multiple fields defined over a 2D domain; the problem of effectively visualizing multiple fields defined over a 3D domain is even more challenging, and an important area for future work. The "Classifying and Combining Visualization Techniques" sidebar presents previous and related work.

\section{Dual vector fields}

While it would be possible to compare the orientation between two vector fields by deriving a scalar field based on the dot product of corresponding vectors, it's also critical for our applications to understand the local magnitude, direction, and gradients of each vector field independent of the other. For this reason, we consider techniques for the multiple representation of $2 \mathrm{D}$ vector fields.

Considering one sample from each of the three visually distinct methods for vector field visualization - texture, glyph, and line (discussed in the sidebar)—we explore the range of effects achievable using layered combinations of these approaches to visualize two colocated vector fields (see Figure 1 on page 76). For the texture-based sample, we use line integral convolution (LIC) — the most widely used texture-based approach. For the glyph-based sample, we create an image by repeatedly texture mapping a comet-like glyph along thick, equally spaced streamlines. This results in a glyph sequence that is continuous in the direction of the flow. We create the linebased sample using the source code supplied by the authors of an equally spaced streamline technique. ${ }^{1}$

One primary goal for each application is to depict the key physical structures of one vector field in the context of the key physical structures in another. Therefore, a highly desirable ability is to easily differentiate between the visual representations of each field and to identify each distribution. This process becomes more complicated when we apply the same representational technique (for example, texture, glyph, or line) to each field. While we could use a supplemental visual variable, such as color, to distinguish the two representations, we 


\section{Classifying and Combining Visualization Techniques}

Many techniques exist for 2D flow visualization. We explore how several of these techniques can be classified and combined for the visualization of multiple related vector and scalar fields.

\section{Classification}

Different visual techniques have characteristics that allow different flow components to be more visually salient. ${ }^{1}$ We classify existing $2 \mathrm{D}$ vector field visualization techniques into one of three visually distinct categories: texture, line, or glyph based.

Texture-based techniques are characterized by a consistent, highly detailed, and dense representation of a vector field. Many texture-based techniques exist for the visualization of 2D flow, as it allows for easy display and analysis of a vector field's fine details. The pioneering technique of spot noise produces a texture from weighted and randomly positioned spots deformed in accordance with flow direction. ${ }^{2}$ Line integral convolution (LIC) - a widely used techniqueinvolves convolving a white noise input texture along calculated streamlines. ${ }^{3}$ We can resolve ambiguous flow direction in LIC images by animating the texture.

Line-based techniques involve the display of elongated streamlines-segments completely tangent to the vector field. These images are fundamentally different than the images created with texture-based techniques as streamlines can be visually traced over a long distance, and line-based images are more sparse than textures (as empty space exists between the lines). While streamlines give a global sense of flow by depicting paths along the vector field, flow direction is not always apparent.

Glyph-based techniques are characterized by the use of repeatable icons that can express various types of information about the flow, including flow orientation. The most basic technique is the use of hedgehogs (sometimes known as vector plots), which are short line segments or arrows aligned with the flow direction at regularly or randomly distributed locations. Glyphs provide the ability to depict flow direction by using the glyph shape or a luminance ramp. ${ }^{4} \mathrm{We}$ also include in this category techniques that involve placing glyphs along calculated streamlines.

\section{Combining multiple images}

Transparency or layering is one of the most effective methods to portray relationships between overlaying components. Kirby, Marmanis, and Laidlaw introduced a method inspired by the layering techniques used in oil paintings to visualize components of 2D flow data. ${ }^{5}$ Weigle et al. demonstrated a texture-generation technique, based on the layering of oriented slivers, using orientation and luminance to encode multiple overlapping scalar fields. ${ }^{6}$ Hotz et al. vary the input texture density and spot size in addition to kernel length and overlay sparse LIC images to visualize tensor field features. ${ }^{7}$

Wong et al. developed a technique that combines elements of alpha channel manipulation, filigreed graphics, and elevation terrain mapping, along with colormap enhancement to visualize a multivariate climate data set. ${ }^{8}$ Our research is inspired by these techniques, and seeks to expand the applications of layering in the visualization of multiple related vector and scalar fields.

\section{References}

1. D.H. Laidlaw et al., "Comparing 2D Vector Field Visualization Methods: A User Study," Trans. Visualization and Computer Graphics, vol. 11, no. 1, 2005, pp. 59-70.

2. J.J. van Wijk, "Spot Noise-Texture Synthesis for Data Visualization," Proc. Siggraph, ACM Press, 1991, pp. 309-318.

3. B. Cabral and L. Leedom, "Imaging Vector Fields Using Line Integral Convolution," Proc. Siggraph, ACM Press, 1993, pp. 263-269.

4. R. Wegenkittl, E. Gröller, and W. Purgathofer, "Animating Flowfields: Rendering of Oriented Line Integral Convolution," Proc. IEEE Computer Animation, IEEE CS Press, 1997, pp. 15-21.

5. R.M. Kirby, H. Marmanis, and D.H. Laidlaw, "Visualizing Multivalued Data from 2D Incompressible Flows Using Concepts from Painting," Proc. IEEE Visualization, IEEE CS Press, 1999, pp. 333340.

6. C. Weigle et al., "Oriented Sliver Textures: A Technique for Local Value Estimation of Multiple Scalar Fields," Graphics Interface, Lawrence Erlbaum Assoc., 2000, pp. 163-170.

7. I. Hotz et al., "Physically Based Methods for Tensor Field Visualization," Proc. IEEE Visualization, IEEE CS Press, 2004, pp. 123-130.

8. P.C. Wong et al., "Multivariate Visualization with Data Fusion," Information Visualization, vol. 1, nos. 3-4, 2002, pp. 182-193. would prefer to reserve the use of color for the communication of related, colocated scalar quantities.

The problem of differentiating between two vector fields is illustrated in Figures 1a, 1b, and 1c. The combination of two texture-based techniques (see Figure 1a) provides a rich representation in which both vector fields are often visible, particularly in regions where the vector field orientations are not aligned. However, it becomes challenging to distinguish the two data sets in regions where the orientations are aligned, as it's difficult to determine which field is being represented by which texture at any given location. Both the glyph and line techniques use an inherently sparse streamline spacing that leaves empty space in the image. Combining two sparse techniques creates a large amount of nega- tive space, which can result in a lost opportunity to represent the vector fields' fine details.

When two glyph-based techniques are overlaid (see Figure 1b), maintaining good visual continuity or good continuation in the flow direction in each field becomes a challenge, particularly when the glyphs are separated by a nontrivial amount of empty space. Overlaying two linebased techniques (see Figure 1c) maintains the continuity of streamlines; however, the accidental patterns of intersections between streamlines in different vector fields can lead to visual artifacts that might cause an inaccurate perception of the data (see Figure 2 on the next page).

Combining different visualization techniques allows for the ability to easily identify and distinguish each distribution (see Figures 1d, 1e, and 1f). When we use a 

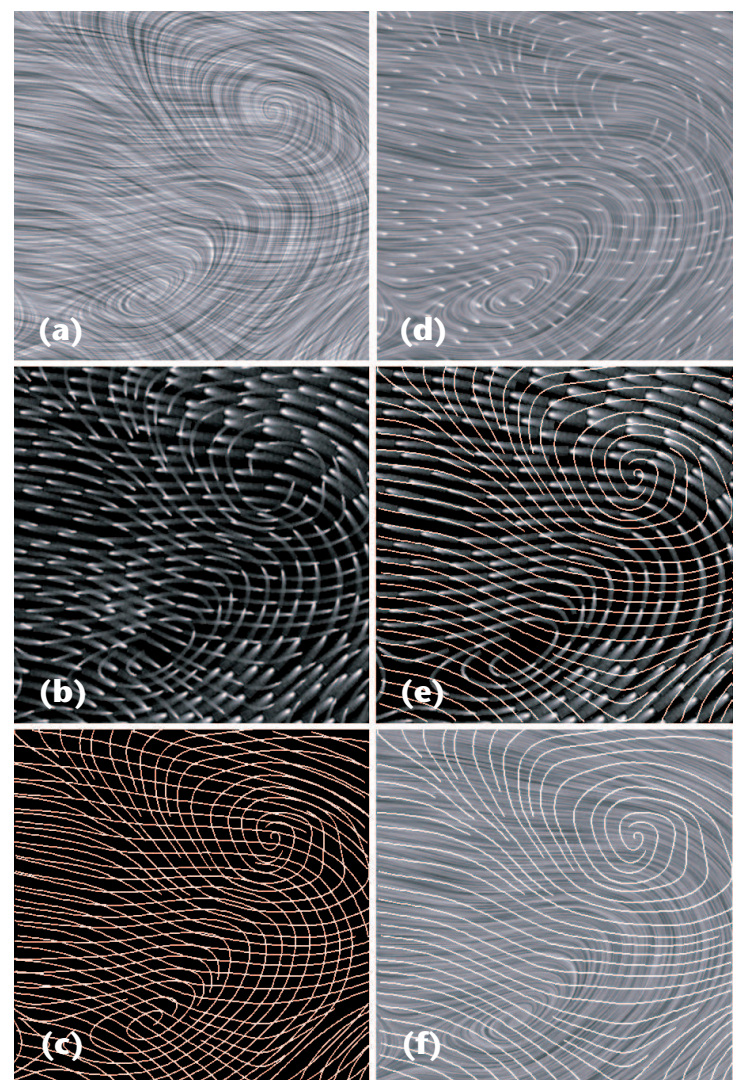

1 Different 2D vector visualization techniques applied to two colocated vector fields: (a) combination of two texture-based techniques, (b) two glyph-based techniques overlaid, (c) two line-based techniques overlaid, (d) glyph- and texture-based techniques overlaid, (e) line- and glyph-based techniques overlaid, and ( $f$ ) line- and texture-based techniques overlaid.

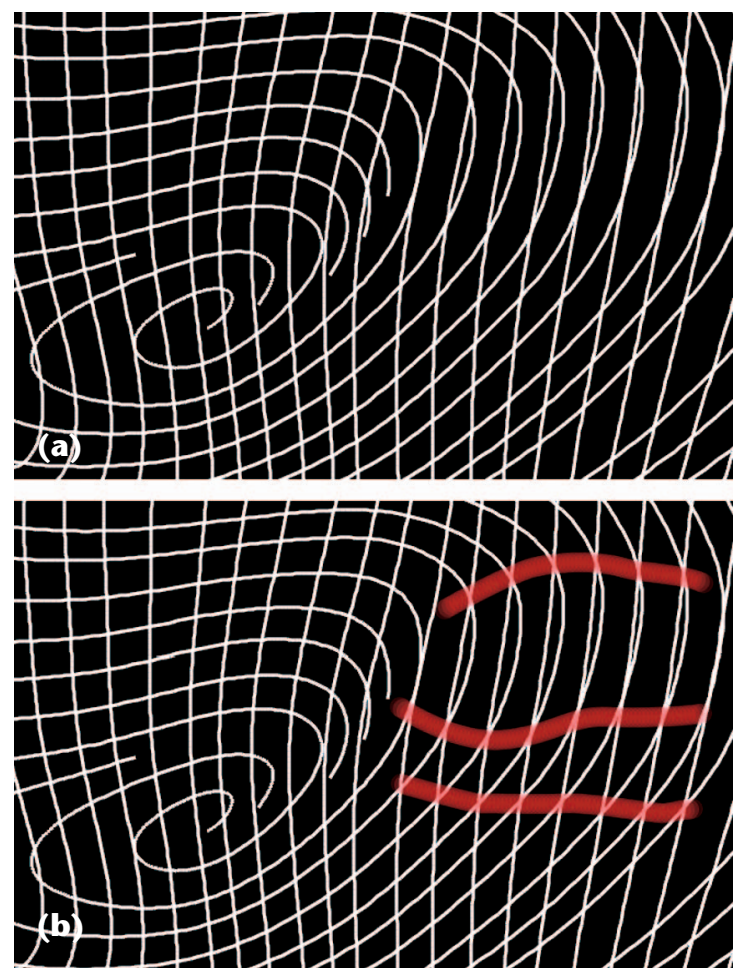

different representational approach for each field, the primary challenge is to appropriately balance the visual prominence of each representation, so that neither overwhelms the other in the combined presentation. Figure 3 illustrates how we can make different representations more or less prominent by altering the contrast and luminance values prior to layering the two images. Similarly, we can use differences in line thickness to make one vector field more prominent when displayed with another (see Figure 4).

In our experience, we can achieve particularly good results by layering a relatively sparser, higher contrast, glyph- or line-based representation of one field over a relatively denser, lower contrast, texture-based representation of the other field. The high contrast between the glyph or line elements and the background or empty space enables them to maintain their visibility when superimposed over the textured background; the glyph or line elements' relatively sparse distribution in the top-layer flow helps enable the simultaneous, effortless appreciation of the flow that the underlying layer portrays.

Next we illustrate methods that allow for two different representations to be combined in such a way that each representation remains distinct and visually separable.

\section{Overlay}

In Figure 5, we've combined two representations using a screen overlay method. This method is similar to a standard image multiplication operation but operates on the additive inverse of each input image. Specifically, if $D(x, y)$ is the output image and $A(x, y)$ and $B(x$, $y)$ are the respective input images, and we assume that each pixel intensity is between 0.0 and 1.0, we define the screen overlay operation as $D(i, j)=1.0-(1.0-$ $A(i, j)) *(1.0-B(i, j))$.

Applying this formula allows the underlying texture image's intensities to show through in the places where the overlying glyph image contains empty (black) space. This technique is most effective when using an image with a black background as the resulting image is highlighted only in regions where the glyph or line is placed. In Figure 5, you can easily distinguish the two vector fields not only where their orientations are nearly orthogonal, but also in the places (such as the upper right corner) where their orientations are nearly aligned. It's precisely to enable these sorts of comparisons of the relative alignments of the two fields that we pursue investigating these methods for their combined portrayal.

\section{Embossing}

In the overlay method, we primarily rely on luminance differences to distinguish the overlying glyph or line elements from the underlying texture image. Embossing is an alternative technique that we can employ to distinguish an overlaid image from an underlying image. ${ }^{2}$ In this method, we give each element of the overlaying image a distinct $3 \mathrm{D}$ visual appearance to enable the elements to perceptually group preferentially with each other and at the same time jointly segregate from the background.

Embossing algorithms simulate a standard lighting equation with a single-point light source. While this method is highly effective in many situations, artifacts 

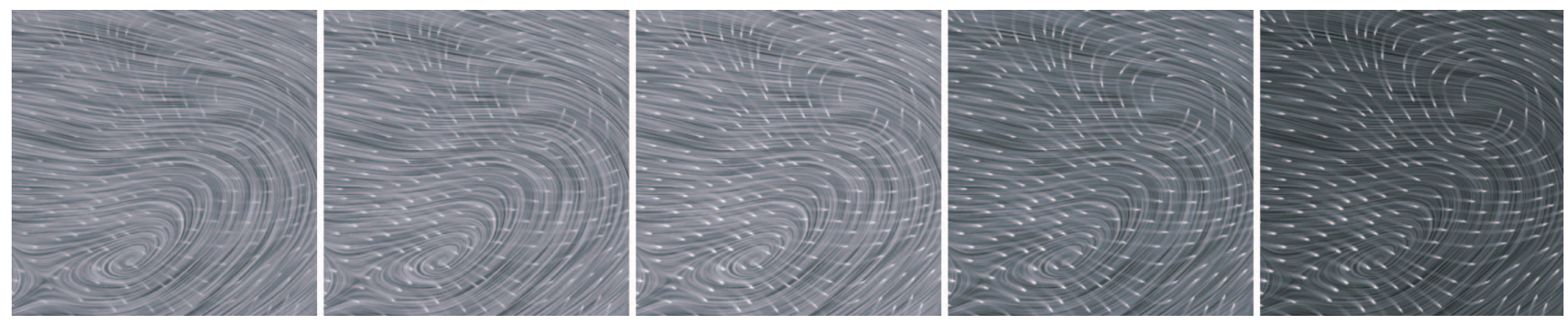

3 We can emphasize different representations by altering luminance properties of individual representations prior to image compositing. In this sequence of images, from left to right, the glyphs become brighter while the texture becomes darker. When the glyphs are subtle (left image), the vector field represented by the texture is more prominent. When the contrast between the white glyphs and the darkened texture is more obvious (right image), the vector field represented by the glyphs is more prominent.

can arise when attempting to emboss vector fields, particularly when the vectors are oriented in the same direction as the light source. To sidestep this problem, we implemented the embossing using two different lights in the plane: one from a source directly above the image, and one from a source above and to the right of the image-that is, at a 45-degree angle. The combination of these directions gives the impression of a broad light coming from above. This produces the visual effect of the embossed image being raised from the surface. We create the composited image on a pixel-by-pixel basis by sampling from the appropriate input image, depending on the flow orientation at each point (taking care to always sample from an image created using a light source direction not aligned with the vector field). Regions of transition are alpha blended between the two images (see Figure 6 on the next page).

Where it's relatively straightforward to emboss streamlines and glyphs, dense textures typically do not emboss well. Embossing is most effective when applied to sparse textures that contain a sufficient amount of empty space within the image. This allows us to portray the shading equation's result (see Figure 7).

We can overlay an embossed image on another image by adding the intensities in the two images on a pixelby-pixel basis and then subtracting the value that the background color takes in the embossed image. This is equivalent to increasing the intensity in the nonembossed image at the points where the intensity in the embossed image is above average, and decreasing the intensity in the nonembossed image at the points where the intensity in the embossed image is below average (see Figure 8). The embossing, in effect, gives a visual distinction of an applied 3D lighting equation and is visually separable from the nonembossed image.

As the initial results of layering a relatively sparse (glyph-like or streamline representation) of one vector field over a denser texture-based representation of the other vector field appear particularly promising, we use this approach to demonstrate the effective representation of two different, related vector fields in three scientific applications.

\section{Application 1: experimental turbulent flow}

Stereoscopic particle image velocimetry (PIV) can help experimentally measure instantaneous compo- nents of a velocity field in a turbulent boundary layer plane in a moderate to high Reynolds number flow. In addition to the experimentally generated velocity field (v), we can numerically calculate and analyze the vorticity vector field ( $\mathbf{w}=\nabla \times \mathbf{v})$ with the velocity vector field. While this data is sampled on a 2D plane, the resulting multivariate data's footprint gives insight into the 3D flow's structure and behavior. Because the knowledge discovery process related to this application is
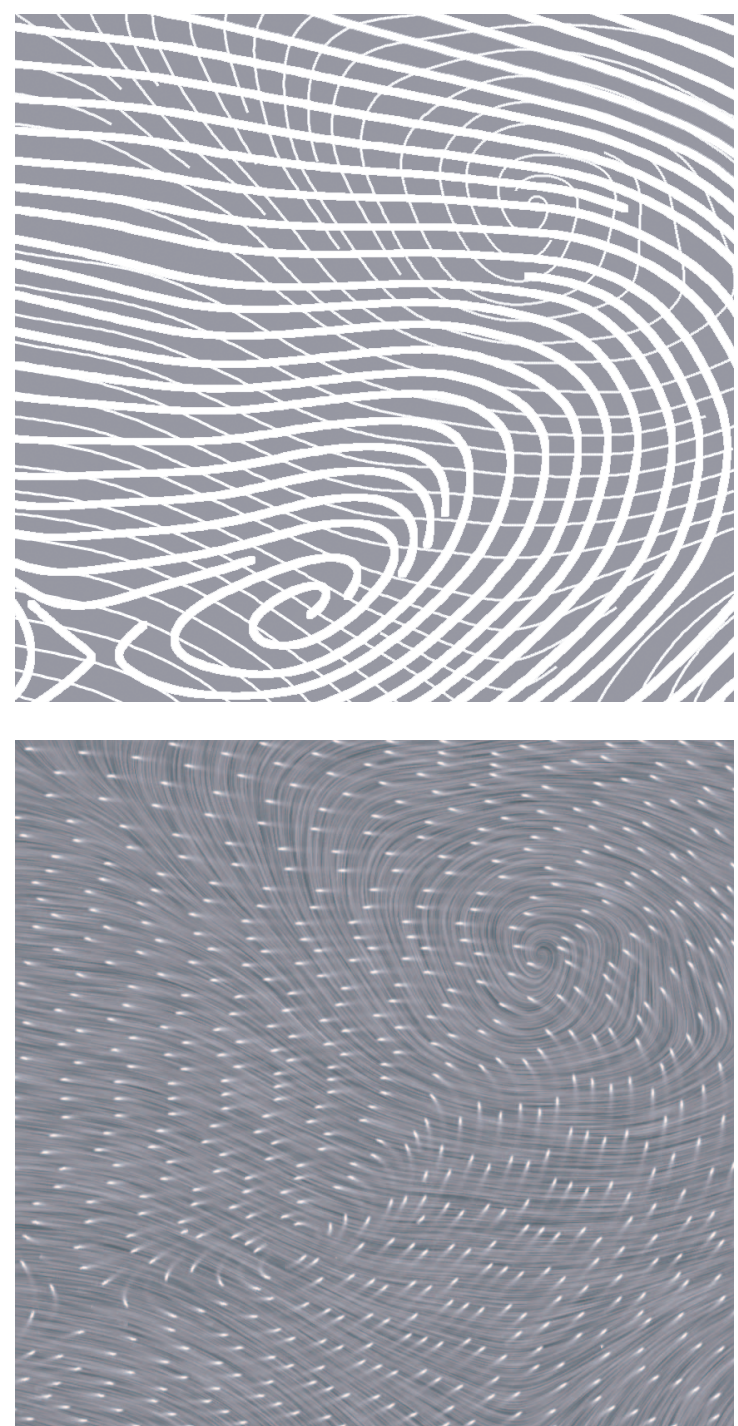

4 Differences in line thickness cause one vector field to appear more prominently than the other.

5 Glyph texture mapped streamlines overlaid on a line integral convolution texture mapped background. 

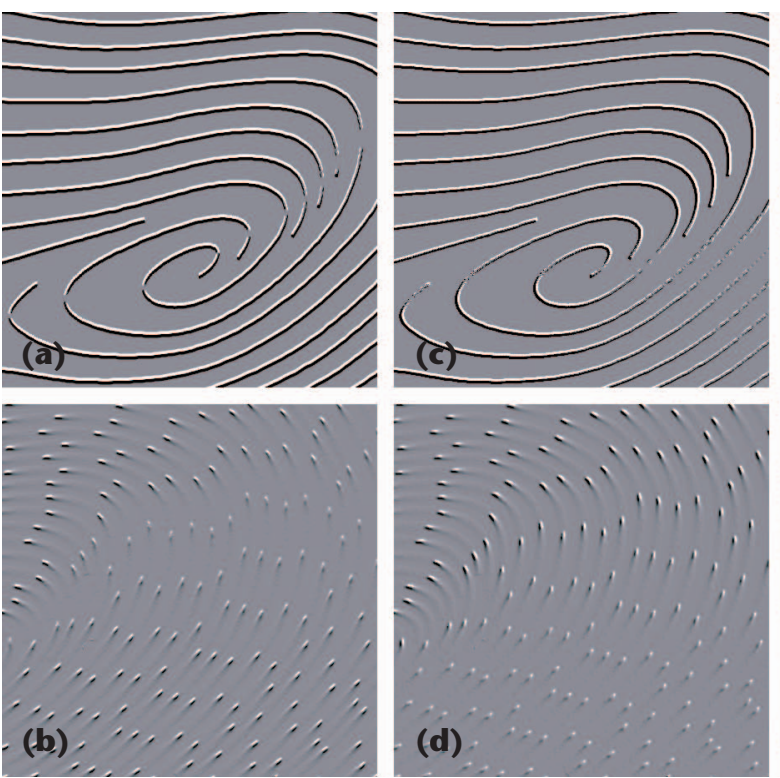
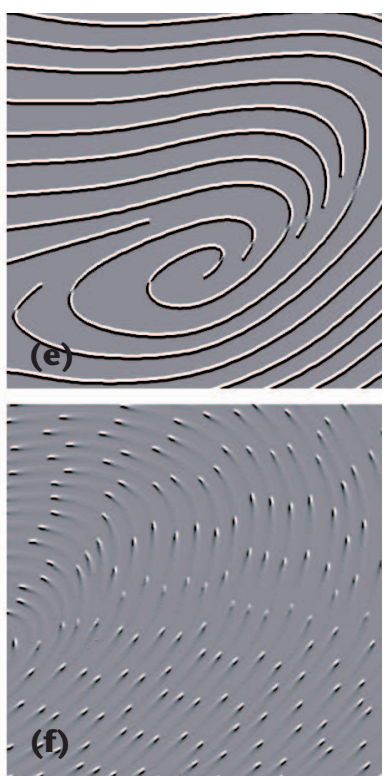

6 Embossing with different light source angles on streamlines (top row) and glyphs (bottom row). (a-b) Light source from directly above (90 degrees). Vectors vertically oriented aren't illuminated well. (c-d) Light source from 45 degrees. In this case, the representation of the diagonally oriented vectors suffers. (e-f) Both light sources combined.

\section{Embossing a} texture. (a) A sparse texture in which the effects of an embossing algorithm can be perceived.

(b) An embossed sparse texture combined with a line integral convolution texture.

8 Distinguishing different fields by embossing. (a) Embossed texture composited with glyphs.

(b) Embossed streamlines composited with texture. (c) Embossed glyphs composited with texture.
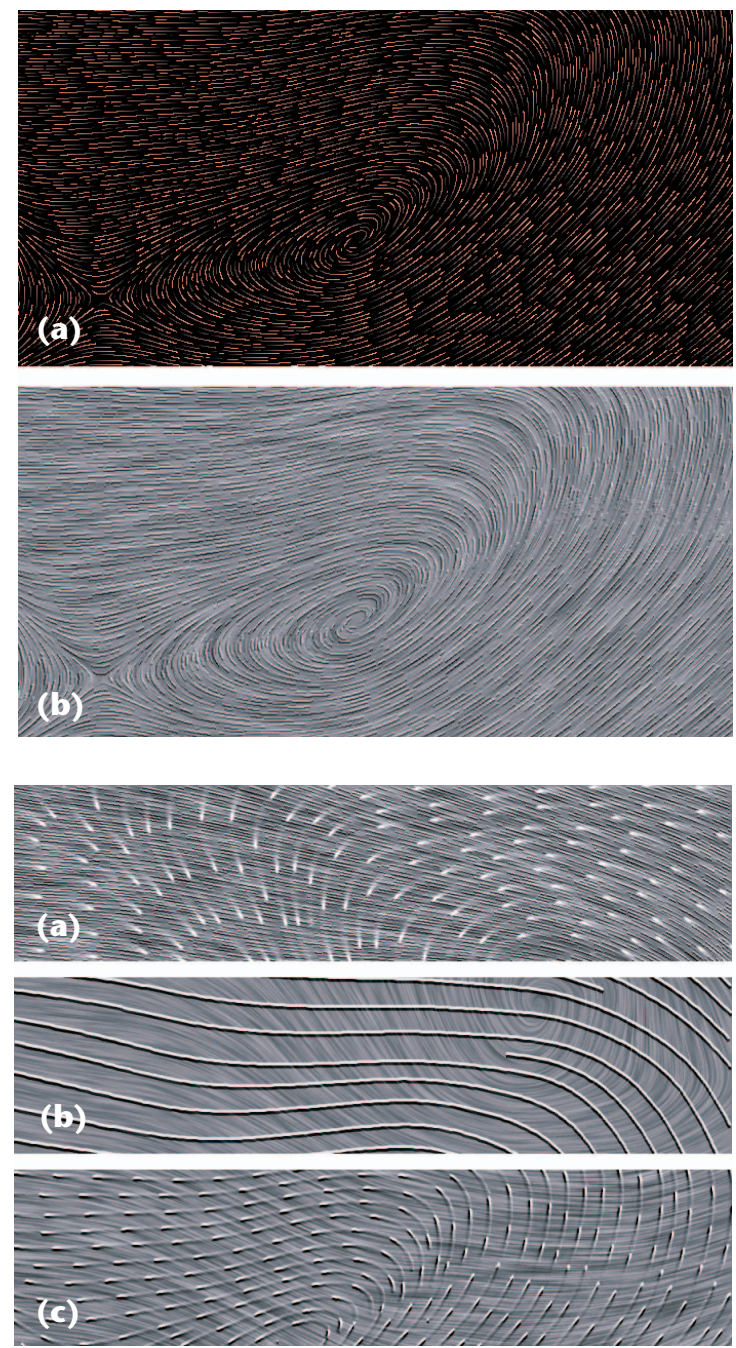

predicated on achieving an integrated understanding of each variable's individual contribution and how the variables interrelate, developing effective, multivariate visualization methods is critically important to the understanding and analysis of PIV experiment results.

Many investigators are interested in understanding the coincidence of the velocity and vorticity fields, particularly in conjunction with the visualization of vortex core locations. These visualizations allow for the analysis of several terms such as $(\mathbf{v} \times \mathbf{w})$, the Lamb vector, and $(\mathbf{v} \cdot \mathbf{w})$, which is proportional to helicity, both of which researchers have suggested as possible vortex tube descriptors. ${ }^{3}$ By visualizing multiple quantities, we can better develop theories about the integrated system, not just individual components of the data.

We use the methods described here to visualize data acquired from experimentally generated dual plane PIV experiments. Our goal is to further understand the relationship among the vorticity vector field, velocity vector field, and 2D and 3D swirl strength scalar distributions.

\section{Swirl strength}

To understand and identify the subtle components of fluid dynamics, researchers have developed several vortex identification methods. Zhou et al. suggested the use of the imaginary part of the complex eigenvalue $\left(\lambda_{\mathrm{ci}}\right)$ of the velocity gradient tensor to visualize vortices. ${ }^{4}$ This value is referred to as the local swirl strength of the vortex. We can compute a simplified version of swirl strength using only in-plane velocity gradients to identify vortex cores. This quantity is referred to as $2 D$ swirl strength and is limited to identifying vortex cores oriented in a position normal to the sampling plane. Dual plane PIV is an extension of single plane PIV in which velocity components are measured in two planes simultaneously. This allows us to calculate all 3D velocity gradient measurements. The corresponding 3D swirl strength is a measure of the existence of a vortex core in any orientation.

We can use the size, strength, and orientation of vortex cores, which can be elucidated from vorticity and 2D and 3D swirl, in theories designed to reduce skin-friction drag in turbulent flow.

\section{Vorticity, velocity, and swirl strength}

We first analyze the in-plane velocity vector along with the 2D and 3D swirl fields. We visualize the velocity vector field using a LIC texture and encode the $2 \mathrm{D}$ and 3D swirl variables in color using a technique that maps each color to alternating streamlines to avoid the ambiguity that occurs when colors are mixed. ${ }^{5}$ The LIC texture serves as a method to convey the underlying vector field while simultaneously providing the means to distribute the color representing additional scalar fields. 
We obtain the velocity vector field by subtracting the mean streamwise velocity from the in-plane velocity component. Thus, the locations of critical points are subject to the relative velocity of the observer and are not germane to the analysis in this example. Swirling streamlines in the vicinity of the area of high swirl strength will only occur in the vicinity of the particular vortex cores that convect at a rate similar to the mean streamwise velocity.

Analyzing the relationship between the 3D swirl and 2D swirl components leads to a valuable understanding of the orientation of a vortex core. As 3D swirl is calculated using all gradient components, it can accurately measure the existence of a vortex core at any orientation to the referencing plane. The $2 \mathrm{D}$ swirl distribution measures only the swirl of a vortex core with significant orientation orthogonal to the plane. Accordingly, 2D swirl is present only where there is also significant 3D swirl.

To develop an understanding of how the vorticity vector field contributes to the phenomenon of swirl strength and the existence of a vortex core, we screen overlay the velocity vector field LIC image with a glyph image representing the vorticity vector field (see Figure 9). Using the result of this image, we can analyze the relationships between the velocity and vorticity vector fields in a way that lends insight to how these two components interrelate.

The glyphs' thickness is directly proportional to the in-plane vorticity magnitude. The two vector fields displayed in Figure 9 reveal zones where velocity and vorticity are nearly perpendicular (regions within a square) and nearly parallel (regions within an oval) for the particular convection velocity being visualized. The region in the center of the image exhibiting strong 2D swirl (red) also contains strong in-plane vorticity, as evidenced by thick glyphs, and additional 3D swirl (blue), suggesting a vortex core inclined at a significant angle to the measurement plane. The remaining zones of 3D swirl that lack a prominent indication of 2D swirl represent cores whose vorticity is more closely aligned with the measurement plane. The glyphs give the core's predominant vorticity direction.

The integrated visualization of these components allows us to correlate the vorticity direction and magnitude with the direction of a vortex core delineated by swirl strength. Effectively combining techniques for multiple vector and scalar field visualization allows for a better understanding of vortex frequency, strength, and orientation, as well as the 3D interactions among and caused by vortices. This technique can be particularly useful in the analysis of large fields characterized by the occurrence of multiple interactions.

\section{Application 2: astrophysical jets}

The modeling of magnetohydrodynamic light supersonic jets in the context of astrophysical galaxy clusters is an area of active research in computational physics. ${ }^{6}$ In addition to the velocity field, physicists are concerned with the magnetic vector field advected by these supersonic jets and with analyzing the relationship between both vector fields. Developing a deeper understanding of the relationship between the vector fields' topology, magnetic field strength, and corresponding velocity structures results in a greater understanding of how kinetic and magnetic energy distributions evolve in these systems and contributes to the explanation of radio emissions that can be physically observed.

In addition to understanding the relationship of the topology of both vector fields, astrophysicists are interested in the interplay between magnetic field strength and the corresponding velocity structures as magnetic field enhancement naturally results from shear and compression in the flow.

\section{Magnetic induction}

Given a magnetic field $\mathbf{B}$, and a vector field $\mathbf{v}$, we can calculate changes in magnetic field from the ideal magnetic induction equation:

$$
\frac{\partial \mathbf{B}}{\partial t}=\nabla \times(\mathbf{v} \times \mathbf{B})
$$

Equation 1 describes how motions of a perfectly conducting fluid change the magnetic fields contained therein. By examining this vector's magnitude, we examine correlations between increases in magnetic field strength and anticipated shear or compression regions in the velocity field. Simultaneous visualization of all velocity field components and the rate of change of the magnetic field strength enables us to locate 


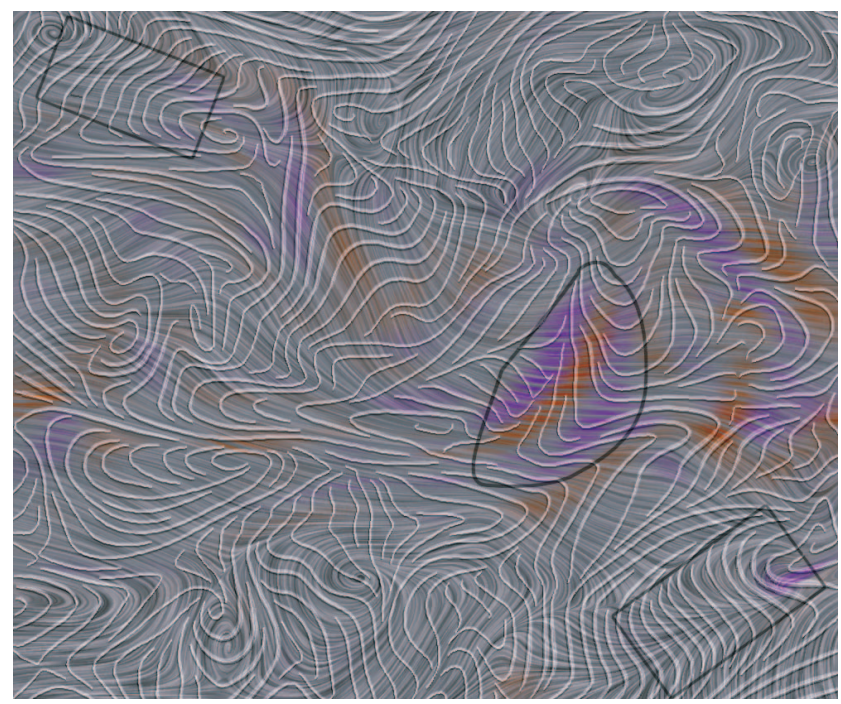

10 Visualization of multiple scalar and vector fields within a magnetohydrodynamic light supersonic jet. The underlying line integral convolution texture depicts the velocity vector field. The embossed streamlines represent the magnetic vector field. Purple (negative) and orange (positive) colors represent the magnitude of the magnetic field's rate of change. The circled area in the center is a region of significant magnetic field amplification where the magnetic field lines change orientation to be oriented perpendicular to the flow. The regions within the rectangles highlight an orthogonal correlation between flow structures and magnetic field lines.

regions of active field enhancement, distinguish newly magnetized fields from those advected along with the plasma, and identify velocity structures that generate enhanced fields.

\section{Multivariate visualization}

Through simultaneous visualization of the simulated velocity and magnetic fields, we have identified several regions of magnetic field enhancement and their antecedent velocity structures. We first visualize the velocity field using a high-frequency LIC texture with the magnitude of the magnetic field's rate of change represented in color. Next, we apply the magnetic vector field's streamlines using the embossing technique introduced previously. We visualize the magnetic field's magnitude using the line thickness. Figure 10 shows the result.

Careful analysis of Figure 10 reveals regions of high magnetic field strength obviously correlated with particular velocity structures such as high-speed flows, flow compression, and shearing between flow structures. Areas in which there exist positive regions of magnetic induction

$$
\left(\frac{\partial \mathbf{B}}{\partial t}\right)
$$

indicate regions of magnetic field amplification. Regions of alignment of the magnetic vector field and the velocity field result from shear enhancement of the magnetic field. There are, however, additional magnetic enhancements with which the velocity field is not obviously causally connected. Moreover, in many instances the magnetic field is unaligned or even orthogonal to the velocity field. This observation runs contrary to theoretical expectations, assuming shear is the dominant form of magnetic field amplification.

Astronomers find an understanding of magnetic field amplification processes and their relationship to flow velocity valuable because these magnetic structures are responsible for the observed radio emission characteristic of these systems. Through simulations and advanced visualization techniques, we expect to learn more about what observations of radio galaxies can tell us about the physics governing their evolution.

\section{Application 3: direct numerical simulation of turbulent channel flow}

Fluid dynamics researchers are interested in modeling turbulent channel flow because the simulation allows for the analysis of large, energetic features in the flow and correlation of flow structures in different layers within the 3D model. Developing a deeper understanding between the different regions within a channel flow results in a better understanding of the formation of vortical structures and the key physical features that cause skin-friction drag. In this application, we consider wall-bounded turbulent flow data from a direct numerical simulation (DNS) of the full Navier-Stokes and continuity equations. ${ }^{7}$

\section{Numerical simulation}

The great value of DNS data is that it's fully resolved and provides full 4D (space and time) data. However, the difficulty with DNS is that it's expensive and limited in Reynolds number. This is because the cost of simulating wall-bounded turbulent flows scales nominally with $R e_{\tau}^{3}$, where $R e_{\tau}$ represents the importance of inertia relative to viscous forces. Thus, larger, faster flows have higher $R e_{\tau}$. The simulation involved 2.7-billion grid points $(3,072 \times 2,304 \times 385)$, with each resulting time step producing a raw (unprocessed) field of 9 Gbytes. The Reynolds number for the simulation is $R e_{\tau}=934$.

The numerical technique involved the integration of the Navier-Stokes equations in the form of evolution problems for the wall normal vorticity and the Laplacian of the wall normal velocity. For spatial discretization, Chebychev polynomials were used in the wall normal direction, while dealiased Fourier expansions were used in wall parallel planes. A third-order, semiimplicit Runge-Kutta scheme was used for temporal discretization. Because a large streamwise extent is needed to capture the longest energy containing motions of the flow, the simulation employed a computational domain of $8 \pi \delta$ units in the streamwise direction and $3 \pi \delta$ units in the spanwise direction, where $\delta$ is the channel half width. 


\section{Regions of flow}

DNS data allows for the investigation of large-scale energetic features and how these might interact between different regions in the flow. Conventional approaches describe wall turbulence in terms of three predominant regions: an inner region, immediately next to the wall, dominated by viscous processes; an outer region, far from the wall, with negligible viscous effects; and an intermediate region, the overlap of the inner and outer regions. This middle region is also referred to as the log region or the inertial wall region. Most existing theoretical approaches regard the inner region to scale universally with inner-wall viscous variables and to be independent of the log region and beyond. Recent studies, however, have shown evidence that the inner region is influenced by outer region parameters. ${ }^{8}$

PIV experimental studies have consistently shown the $\log$ region to contain packets of hairpin vortices, with long streamwise regions of momentum deficit and with high-speed fluid seeming to fill the separation between neighboring motions. However, the PIV fields are somewhat limited in length (approximately $2 \delta$ ). Researchers have recently discovered that the structures can exist up to $20 \delta$ in length and may have a tendency to meander in the spanwise direction. ${ }^{9}$ The tendency of the lowspeed region to meander across the flow explains why such long length scales have not been observed previously from single-point measurements in boundary layer flows.

\section{Visualization of multiple layers}

The discovery of the long superstructures is particularly significant as it indicates an outer-layer scaled phenomena might have influence all the way down to the wall in the inner layer. The DNS data is useful for investigating this conjecture; Figure 11 shows some results.

Figure 11 shows two planes simultaneously from the DNS data set. The embossed glyphs data represents a plane in the log region $\left(z^{+}=150\right)$ and the underlying texture data is in the viscous buffer zone $\left(z^{+}=15\right)$ at the wall normal location of maximum turbulent energy production. The luminance values for the texture and the glyph size show the instantaneous streamwise values for each plane. Dark, long, low-speed structures are visible in the viscous buffer zone, and careful examination indicates that the footprints of these structures extend to the log region based on the presence of large glyphs in coincident locations. The long superstructures described previously are approximately outlined in red. These results indicate that near-wall regeneration mechanisms are not independent of the slow dynamics associated with structures on the order of the external dimension of the flow, as has always been previously believed.

\section{Discussion and conclusion}

We have explored the development of visualization methods that can provide a deeper and more comprehensive understanding of the key interrelated features in coincident vector fields, augmented with the simultaneous display of related coincident scalar quantities. The needs of fundamental fluids research problems have driven our investigations: investigations by aerospace engineers into the physics of turbulence, and the computational modeling and simulation by astrophysicists of the behavior of supersonic jets in galactic clusters. In each of these application areas, we have demonstrated how our developed techniques facilitate the process of scientific inquiry.

This interaction between visualization researchers and domain scientists has proven extremely beneficial to both parties. Through our collaboration we have advanced our ability to create effective visualizations and made important discoveries that further the development of important theories related to the applications. The application scientists played a critical role in defining the specific needs that the visualization techniques presented here were developed to address. In addition, they provided an objective assessment of the functionality of the methods, in terms of how well they meet their goals of gaining greater insight into their data.

The focus of our research so far has been the exploration of different visualization methods, and we have not yet honed our code into a production quality tool. However, we eventually intend to package our various utilities into a user-friendly application - using current graphics hardware - that the application scientists can use on a routine basis for the analysis of coincident multiple vector fields. Future work will also include expanding our investigations to consider a wider variety of texture patterns beyond LIC. Finally, we are also considering techniques to explore the potential effectiveness of various alternative methods for simultaneously portraying multiple colocated vector and scalar fields in 3D.

\section{Acknowledgments \\ We thank Bharathram Ganapathisubramani for the collection of the dual plane PIV data, Robert Moser for}


the use of the DNS data, and Nicholas Hutchins for his analysis and assistance with the DNS data. We also thank the anonymous reviewers for their valuable comments and suggestions. This research was supported by the Digital Technology Center at the University of Minnesota and by grants from the US National Science Foundation (AST03-07600, CTS-0324898, ACI-9982274) and the David and Lucile Packard Foundation.

\section{References}

1. G. Turk and D. Banks, "Image-Guided Streamline Placement," Proc. Siggraph, ACM Press, 1996, pp. 453-460.

2. T. Urness et al., "Techniques for Visualizing Multi-Valued Flow Data," Proc. Eurographics/IEEE TCVG Symp. Data Visualization, Eurographics Assoc., 2004, pp. 165-172.

3. H.K. Moffatt, "Magnetostatic Equilibria and Analogous Euler Flows of Arbitrarily Complex Topology. Pt. 1: Fundamentals," J. Fluid Mechanics, vol. 159, 1985, pp. 359378.

4. J. Zhou et al., "Mechanisms for Generating Coherent Packets of Hairpin Vortices in Channel Flow," J. Fluid Mechanics, vol. 387, 1999, pp. 353-396.

5. T. Urness et al., "Effectively Visualizing Multi-Valued Flow Data Using Color and Texture," Proc. IEEE Visualization, IEEE CS Press, 2003, pp. 115-121.

6. S.M. O'Neill et al., "Three-Dimensional Simulations of MHD Jet Propagation through Uniform and Stratified External Environments," The Astrophysical J., vol. 633, no. 2, 2005, pp. 717-732.

7. J.C. del Álamo et al., "Scaling of the Energy Spectra of Turbulent Channels," J. Fluid Mechanics, vol. 500, 2004, pp. 135-144.

8. B. Ganapathisubramani et al., "Investigation of Large-Scale Coherence in a Turbulent Boundary Layer Using Two-Point Correlations," J. Fluid Mechanics, vol. 524, 2005, pp. 5780.

9. N. Hutchins, W. T. Hambleton, and I. Marusic, "Inclined Cross-Stream Stereo PIV Measurements in Turbulent Boundary Layers," J. Fluid Mechanics, vol. 541, 2005, pp. 21-54.

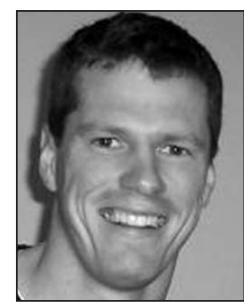

Timothy Urness is an assistant professor in the Department of Mathematics and Computer Science at Drake University. His research interests include developing techniques for the effective visualization of multidimensional flow data. Urness has a $P h D$ in computer science from the University of Minnesota. Contact him at timothy.urness@ drake.edu.

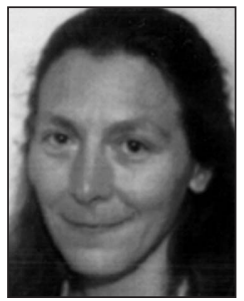

Victoria Interrante is an associate professor in the Department of Computer Science and Engineering at the University of Minnesota. Her research interests include the application of insights from visual perception to the design of techniques for more effectively conveying information through images. Interrante has a PhD in computer science from the University of North Carolina at Chapel Hill. Contactheratinterran@cs.umn.edu.

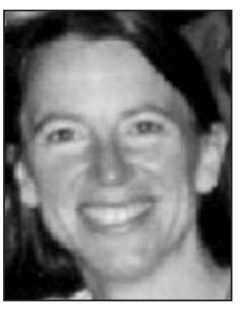

Ellen Longmire is a professor in the Department of Aerospace Engineering and Mechanics at the University of Minnesota. Her research interests include experimentalfluid mechanics including turbulent, multiphase, biomedical, and microscale flows. Longmire has a PhD in mechanical engineering from Stanford University. Contact her at ellen@aem.umn.edu.

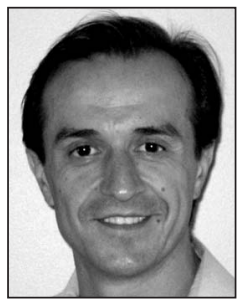

Ivan Marusic is an associate professor in the Department of Aerospace Engineering and Mechanics at the University of Minnesota. His research interests include experimental and theoretical fluid mechanics, with an emphasis on turbulence. Marusic has a $\mathrm{PhD}$ in mechanical engineering from the University of Melbourne, Australia. Contact him at marusic@aem.umn.edu.

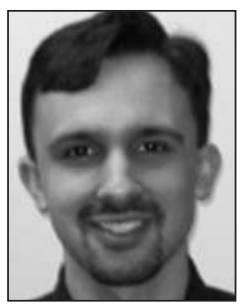

Sean O'Neill is a PhD student in the astronomy department at the University of Minnesota. His research interests include numerical studies in high-energy astrophysics, specifically the simulation and analysis of supersonic jets in realistic galaxy cluster environments. Contact him at soneill@astro.umn.edu.

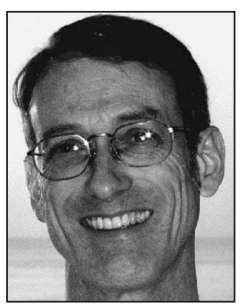

Thomas Jones is a professor of astronomy at the University of Minnesota. His research interests include computational fluid dynamics, highenergy astrophysics, and cosmology. Jones has a PhD in physics from the University of Minnesota. Contact him attwj@umn.edu.

Article submitted: 25 Aug. 2005; revised: 4 Jan. 2006; accepted: 28 Feb. 2006. 


\section{University Library}

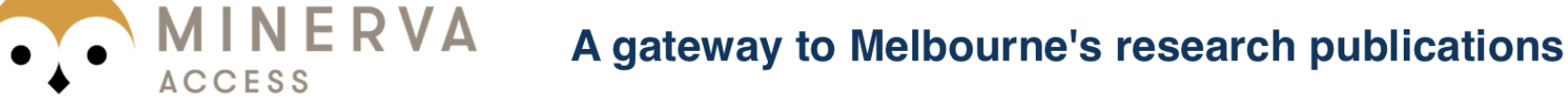

Minerva Access is the Institutional Repository of The University of Melbourne

Author/s:

Urness, T;Interrante, V;Longmire, E;Marusic, I;O'Neill, S;Jones, TW

Title:

Strategies for the visualization of multiple 2D vector fields

Date:

2006-07-01

Citation:

Urness, T., Interrante, V., Longmire, E., Marusic, I., O'Neill, S. \& Jones, T. W. (2006).

Strategies for the visualization of multiple 2D vector fields. IEEE COMPUTER GRAPHICS AND APPLICATIONS, 26 (4), pp.74-82. https://doi.org/10.1109/MCG.2006.88.

Publication Status:

Published

Persistent Link:

http://hdl.handle.net/11343/34778 